\title{
Arrhythmia Classification Using Fractal Dimensions and Neural Networks
}

\author{
Ben Ali Sabrine ${ }^{1, *}$ Aguili Taoufik $^{2}$ \\ 1,2 Communication System Laboratory Sys'Com, \\ National engineering School of Tunis, University Tunis El Manar, Tunisia \\ sabrinebenali05@gmail.com
}

\begin{abstract}
According to statistics, there has been a big increment in death in consequence of failures worldwide. Electrocardiogram was chosen as a possible implement for diagnosing cardiovascular diseases, it is a test that records the electrical activity given by the heart muscle and how it contracts. In this vein, our work is reported to analyze this low-cost and widely available signal. One of major issues that arise during the analysis of the electrical activity in the heart is noise reduction in electrocardiogram signals. The best bothersome noise sources have frequency components within the electrocardiogram spectrum. Thus, noises are difficult to take away using standard filtering procedures. Indeed, we show how wavelets can be used to denoise such signals. For this reason, electrocardiogram signal is considered as a selfsimilar object. As a result, fractal analysis can be used to make better use of the information gathered. The fractal dimension is considered the best explanation of the electrocardiogram signal that can account for its hidden complexity. This paper uses the fractal dimension to introduce a new technique for the simple classification of arrhythmias from electrocardiogram signals. We used neural networks to improve our classification results, as variety is one of the most active research and application areas for neural network
\end{abstract}

Keywords: Electrocardiogram Signal, Fractal dimension, wavelet theory, Classification of cardiac diseases, neural networks

\section{INTRODUCTION}

Heart disease is the important common reason of death worldwide. Therefore, the diagnosis of these dangerous diseases seems a vital task. The statistics point that the increasing number of deaths is due to cardiovascular defaults. The World Health Organization stated that about 17.3 million people died in 2008, caused by this difficulty [1]. This number is predicted to reach 23.3 million individuals by 2030 . Therefore, about $80 \%$ of death cases have emerged in countries with low or not medium-level per person earnings, and most of them have arising from late diagnosis of the disease or misdiagnosis. Since the electrogram (ECG) is one of the most accurate tools and cost-effective for diagnosing the cardiac diseases, the heart comportments can be attentively evaluated using this tool [2]. The electrocardiogram shows the electrical activity given by heart. It brings together a set of waves that are directly correlated with this activity. They are identified by the QRS complex, the $\mathrm{P}$ wave and the $\mathrm{T}$ wave, where the QRS complex reflects ventricular depolarization; the $\mathrm{P}$ wave indicates atrial depolarization and the $\mathrm{T}$ wave reflect ventricular repolarization.

These waves occupy frequency spectra and temporal locations that are generally different for the same signal from the same healthy subject or with cardiac pathology. Analysis of ECG recordings can diagnose and locate a large number of pathologies, but is not sufficient to detect certain abnormalities. For this we need a more reliable technique that allows us to analyze an ECG signal. The objective of this work was the use of wavelet denoising in noise reduction of ECG signals. Especially, the influence of the selection of wavelet function and the choice of rotting level on the denoising process. The ECG signals present the fractal properties. As a result, the fractal dimension, which represents the non-linear 
energy, is the right method for carefully studying and extracting the characteristics from these signals $[3,4]$.

\section{MATERIALS AND METHODS}

The block diagram of the proposed method has been demonstrated in Figure 1. As shown in Figure, the entire methodology is divided into three basic steps: preprocessing, calcul the fractal dimension and classification. In the preprocessing step, the sampling frequencies have been standardized to a base frequency that elevates the speed of the algorithm so the accuracy of the classification step. After, the filtering and Baseline wander removal of electrocadiogram signals were performed based on the wavelet transform. Then, we calculate the fractal dimension. The last step is dedicated to the classification of heart disease. All steps are explained in the following sub-sections

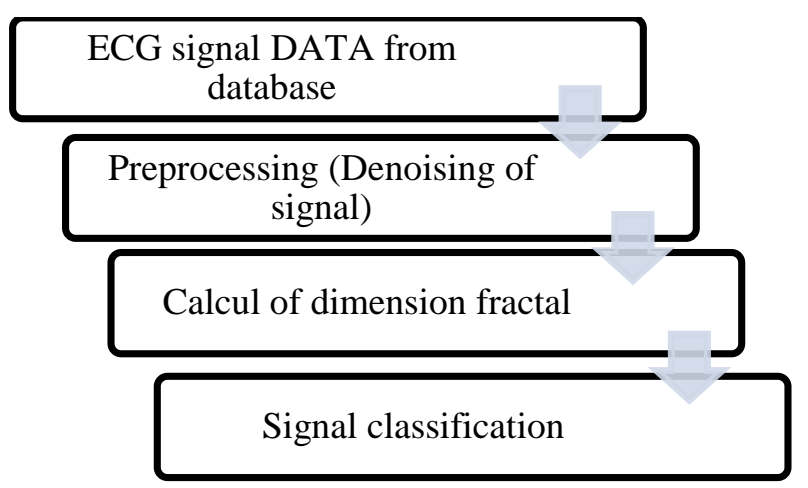

Figure 1 Proposed method of ECG analysis

\subsection{Database}

First, The MIT / BIH physiological database is made up of different databases, the most widely used of which is the basis of MIT / BIH arrhythmia [5]. This database has 48 electrocardiogram signal recordings of 47 different cases. Every recording lasts about 30 minutes, the resolution is 200 samples per $\mathrm{mV}$ and the sampling frequency is $360 \mathrm{~Hz}$. All records include more of 109,000 beats classified into 15 different types of heartbeats. The different pathologies are divided according to the MIT$\mathrm{BIH}$ basis into arrhythmia class. Approximately, the most abundant class is the class of normal rhythms (NOR) with $70 \%$ of the beats.

\subsection{Preprossing}

The preprocessing of the ECG signal consists of sampling the QRS complex and determining the reference point relative to the extremum of this complex (R). Using the list of reference'points of the ECG signals given by the base MIT_BIH. By the reference point (R), it's possible to determine the start $(\mathrm{Q}=\mathrm{R}-50 \mathrm{~ms})$ of the
QRS and its ending ( $=R+100 \mathrm{~ms}$ ) (fig. 2). Thence the QS interval is of the order of $150 \mathrm{~ms}$, or 54 samples for a sampling frequency of $360 \mathrm{~Hz}$ [6]. This pretreatment is performed for each complex labeled as heart disease of type LBBB, P, NOR, RBBB, AP, FPN, FVN and PVC from the MIT_BIH base

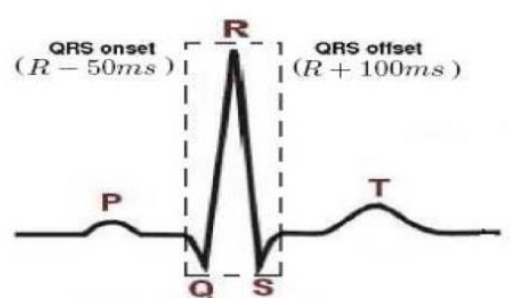

Figure 2 Determination of the QS interval [6].

In this stage, it is indispensable to eliminate noise from input signals. Noise removal in the preprocessing of the ECG signal includes different success plans for each noise source [7] .We will de-noise the ECG signal using the wavelet transform. Selecting the mother wavelet $\psi_{\mathrm{m}, \mathrm{n}}$ (t) is the first step of wavelet denoising procedure, which forms set of functions (family of wavelets).Furthermore, mother wavelet was used:

$$
\psi_{\mathrm{m}, \mathrm{n}}(\mathrm{t})=2^{\mathrm{k} / 2} \psi\left(2^{-\mathrm{k}} \mathrm{t}-\mathrm{n}\right)
$$

\section{$\mathrm{k}$ : coefficient of scale (compression) \\ $\mathrm{n}$ : coefficient of time translation}

The next phase is selecting the number of decomposition levels of signal $\mathrm{x}_{\mathrm{i}}(\mathrm{t})$. As a consequence of this transformation so-called details $(\mathrm{cDj})$ and approximations $(\mathrm{cAj})$ are obtained.

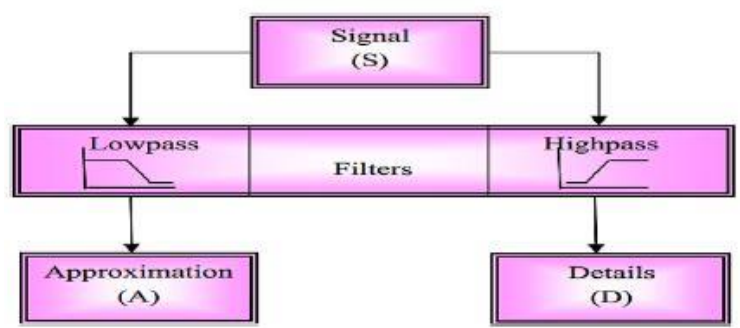

Figure 3. decomposition of signal into detail and approximation[8]

details $(\mathrm{cDj})$ contain high frequency components $\mathrm{t}$ in signal, while approximations $(\mathrm{cAj})$ correspond to low frequency . Next step of decomposition signal $x(t)-$ detail coefficients $\mathrm{cDj}+1$ and approximation $\mathrm{cAj}+1$ are obtained in similar way, when in place of original signal approximation coefficients of $\mathrm{j}$-th decomposition level are studied . 


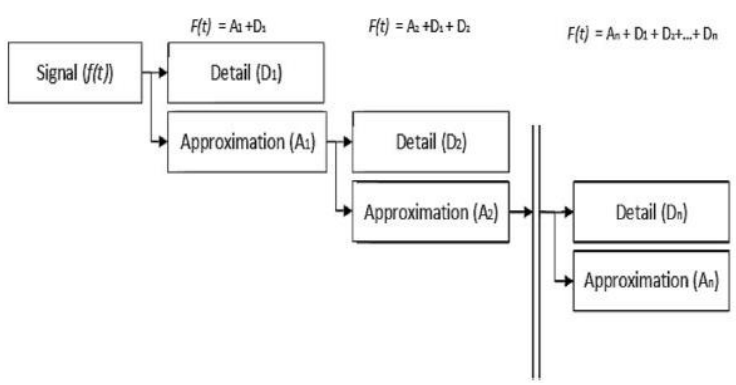

Figure 4. Diagram of multiresolution analysis of signal [8]

The final stage of our analysis is reconstruction of signal $\mathrm{x}_{\mathrm{i}}(\mathrm{t})$ based of approximation coefficients chosen $\mathrm{i}$ th level of decomposition $\left(\mathrm{cA}_{\mathrm{i}}\right)$ and modified detail coefficients from $\mathrm{i}$-th $\left(\mathrm{cD}_{\mathrm{i}}\right)$ as well as higher levels of decomposition:

$\mathrm{x}_{\mathrm{i}}(\mathrm{t})=\sum_{n} C \mathrm{~A}_{\mathrm{m}, \mathrm{n}} \varphi_{\mathrm{k}}\left(2^{-\mathrm{m}} \mathrm{t}-\mathrm{n}\right)+\sum_{m=m 0}^{m L} \sum_{n} C \mathrm{D}_{\mathrm{m}, \mathrm{n}} \psi_{\mathrm{m}, \mathrm{n}}\left(2^{-\mathrm{m}} \mathrm{t}-\mathrm{n}\right)$

Where $(\mathrm{t})$ present scaling function from L-th level of decomposition, $\psi_{\mathrm{m}, \mathrm{n}}(\mathrm{t})$ are wavelet functions for $\mathrm{m}=\mathrm{m}_{0} \ldots \mathrm{m}_{\mathrm{k}}$ levels of decomposition.

the following figure present a sample of ECG signal filtering by this theory

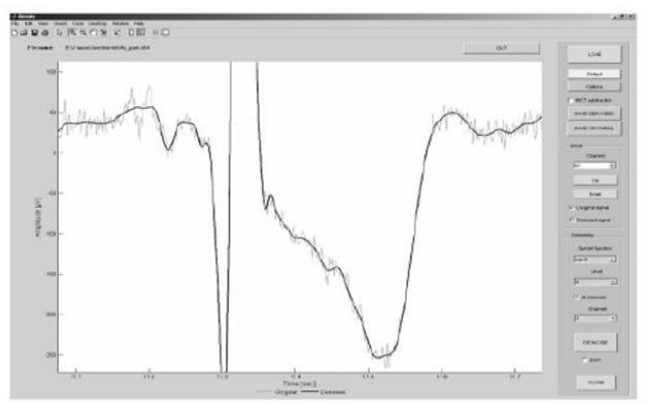

Figure 5. Enlarged fragment of original (grey line) and filtering ECG signal (black line) from single lead is shown.

\subsection{Fractal Dimension}

The fractal dimension is one of the important characteristics of fractal objects. It is considered the universal index of the degree of their irregularity [9]. "If fractal can be expressed as a union of sets, it is strictly self-similar each of which is a just minimal copy of the full set (Koch flack ,Sierpinski triangle). The best fractal looking in nature does not display this exact form. Natural objects are not union of exact reduced copies of whole. A amplified view of one part will not precisely reproduce the whole object, but it will have the same empirical appearance. This property is called semi-selfsimilarity or statistical self-similarity. Like ECG signal of a human heart is a self-similar object,so it must have a fractal dimension.’'[10]

\subsubsection{Methods of calculating the (FD) fractal dimension}

To calculate the Fractal Dimension, there are many algorithms such as Katz [12], box-counting [13], Higuchi [14], Regularization [15], and etc. Each of them has its own advantages and disadvantages. Using the Katz'algorithme for example, the fractal dimension is obtained directly from the time series and can be defined as follows:

$$
\mathrm{DFK}=(\log (\mathrm{L})) /(\log (\mathrm{d}))
$$

Where $\mathrm{L}$ is the (Euclidean) distance between the first point in the series and the point that provides the furthest distance with respecting our first point and the total length of the ECG time series. For waveforms, which are ordered sets of pairs of points (x, y),the length $\mathrm{L}$, is the sum of distances between consecutive points, that is:

$$
\mathrm{L}=\operatorname{sum}(\operatorname{dist}(\mathrm{i}, \mathrm{i}+1))
$$

The dist $(a, b)$ presents the distance between two pairs of points $\mathrm{b}=(\mathrm{u}, \mathrm{v})$ and $\mathrm{a}=(\mathrm{x}, \mathrm{y})$. Monotonically advancing waveforms have natural starting points. So, the diameter of a waveform can be believed as the furthest distance between the initiate point (point 1) and any other point (point i) of this waveform, namely

$$
\mathrm{d}=\max (\operatorname{dist}(\mathrm{i}, \mathrm{i}))
$$

There is also the Hausdorff'algorithme which consists in covering the curve with balls $\mathrm{Bi}$ whose diameter (diam $\mathrm{Bi}$ ) is less than or equal to $\varepsilon$. The sum of the diameters of the balls provides an approximation of the measure of the whole. As the intersections of these balls are not empty, we therefore consider the minimum of these measurements. To obtain the best approximation of $\mathrm{E}$ by these overlaps, we tend $\varepsilon$ towards 0 . The Hausdorff dimension is given by

$$
\mathrm{DFH}=\quad \lim _{\delta \rightarrow 0} \frac{\ln N \varepsilon}{\ln \left(\frac{1}{\varepsilon}\right)}
$$

Where Ne is the minimum number of balls of diameter $\varepsilon$, necessary for paving the curve.

These methods and others have been studied and reviewed by Kourosh Kiani and Farzane Maghsoudi [16]. It has been included that Higuchi algorithm is an appropriate method and one of the best representatives of disease

\subsubsection{Higuchi algorithm}

The Fractal Dimension (FD) is a depictive measure that has been confirmed useful in putting into numbers the complex difficulty or self-similarity of the biomedical signals. ECG signal is a self-similar object; consequently, it must have a fractal dimension (FD) that can be obtained 
through mathematical methods to distinguish and identifying apparently particular states of cardiac disease-related conditions [11]. As we said in the previous paragraph, for calculating the $\mathrm{FD}$, there are many and different algorithms. It has been included that the Higuchi algorithm is an appropriate method and one of the best representatives of disease.

Suppose; time series: $x=\{x(1), x(2), \ldots, x(N)\}$, the FD is calculated thus:

a) formulate $\mathrm{S}$ new time series: $x \mathrm{~ms}$ are defined as:

$$
x \mathrm{~ms}=\{(m), \ldots, x(m+\lfloor(N-m) / \mathrm{S}\rfloor \mathrm{s})\}
$$

With $\mathrm{m}=1,2, \ldots, \mathrm{s}$ indicates the initial time value, and $\mathrm{S}$ represents the discrete time interval among points.

b) Calculate the length of respectively new time series as before defined:

$$
L \mathrm{~m}(\mathrm{~s})=1 \mathrm{~s} /\{(N-1) /[(N-m) / \mathrm{s}\rfloor \mathrm{s} \Sigma \mid x(m+i \mathrm{~s})-x(m+
$$$$
(i-1) \mathrm{s}) \mid\}
$$

With $(N-1) /\lfloor(N-m) / s\rfloor$ represents a normalization factor

c) Calculate the length of curve for the time interval S:

$$
\mathrm{L}(\mathrm{S})=1 / K \Sigma \mathrm{m}=1 \mathrm{k} \mathrm{m}(\mathrm{S})
$$

d) In the end, and according to the consequential equation, D presents the Fractal Dimension (FD) curve

$$
\mathrm{L}(L(\mathrm{~S}))=D \log (1 / \mathrm{S})+\bar{b}
$$

\section{NEURAL NETWORKS}

Scalar variables A neural network is the linkage of foundational objects, formal neurons. The basic networks are distinctive by their architecture, the organization of the graph (complete, in layers,...), its level of complexity (presence feedback loops in the network the neurons 'number,), by neurons' type (their activation or transition functions) and finally by the target: optimization, learning supervised or not, dynamic systems ...

The Neural Networks are improved at identifying trends or patterns in data and they are well appropriate for forecasting or predicting. Therefore neural networks are considerably applied to the biomedical systems. An analysis is performed to prompt neural network (NN) applications in the medical diagnosis. Neural Networks are exploited analytically to model the system of human cardiovascular. Diagnosis can be succeeded by developing a model of an individual's cardiovascular system and comparing it with the real time physiological measures picked up from the patient. If this subroutine is achieved regularly, the potential harmful medical conditions can be determined at an early phase.

We start by locating the QRS, P, and T waves and by calculating the parameters temporal and morphological which characterize a beat using mainly the wavelet technique.

Then we implemented a system analysis and classification of beats cardiac networks based on the combination of supervised learning artificial neurons and unsupervised. This system is responsible for determine the type of beat according to his characteristics [17]

\section{RESULTS AND DISCUSSION}

\subsection{Classification of ECG using fractal dimension}

The ECG signal is considered as self-similar object. So we need using the fractal analysis.

After filtering the ECG signal using the wavelet theory, we calculate the fractal dimension (FD) based on the Higuchi algorithm by Matlab. In this study, the fractal dimension is inured to distinguish between the ECG signals of healthy person and unhealthy persons with Premature Ventricular Complex (PVC), Premature Atrial Contracture (PAC) and Paroxysmal Supraventricular Tachycardia (PSVT) from the raw ECG data. According to the results and with regard to the fractal dimension, the ECG signals can be classified as follows:

Normal: FD >1.56

PAC: $1.37<\mathrm{FD} \leq 1.56$

PVC: $1.3<\mathrm{FD} \leq 1.37$

PSVT: $1<\mathrm{FD} \leq 1.3$

So according to the value of the fractal dimension we can distinguish between a disease and a normal case. In addition we can estimate if it is a PVA, PAC or PVST. So the aim of this work is classifying arrhythmias based on a fractal dimension.

\subsection{Classification ECG based on the neural network}

Figures and tables should be placed either to them if possible. Classification is one of the most widely used techniques in medicine. It is the basic principle of several diagnostic support systems. This involves assigning a set of objects (recordings of electrocardiograms, encephalograms, etc.) to a class according to a descriptor thereof. This description is carried out by means of properties or typical conditions specific to the classes. The objects are then classified according to whether or not they verify these conditions or properties. The 
modifications which appear on the graph of the ECG are often indicating pathologies, from where interest of the analysis of these signals by an automatic system for better exploiting the information recorded during a Holter examination and the identification of the parameters representative of various pathologies. Analysis and the characterization of signal ECG by descriptors constitute a paramount stage for the classification and for establish a diagnosis. We developed in this work a classifier of cardiac arrhythmias by applying neural networks.

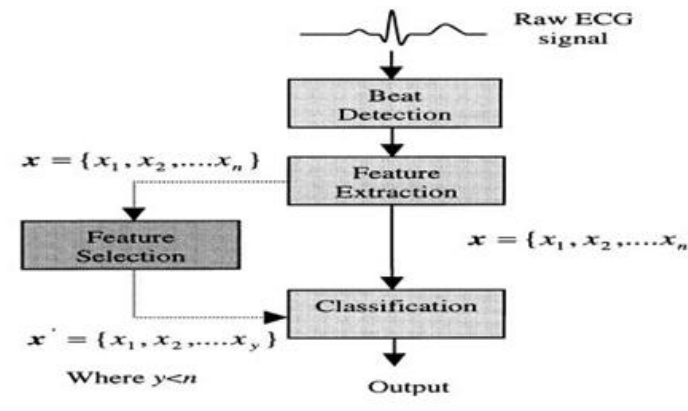

Figure 6 Functional modules in a typical Computerised Electrocardiographic system

'Researchers have used many types of neural network (NN) for classification of electrocardiogram signals. Therefore, artificial neural networks (ANNs) are selfadaptive, data driven, non-linear, accurate and fast. Additionally it is easily scalable and robust to noise. The ANNs have many advantages:

1) It offers non-linear mapping among inputs and outputs through activation function such as sigmoid to solve non-linear problem, for example classification of electrocardiogram (ECG) signals.

2) The ANN can adaptively modelling the lower frequencies of the electrogram (ECG) signal which are essentially non-linear

3) It can carry out better or similar results than deterministic or statistical methods. Statistical approaches performs good for linear problems but it cannot produce best results for non-linear problem, due to the fact that the statistical methods are produced based on the presumption of provided linear time series.' [18]

\section{CONCLUSION}

In this study, the filtration by wavelet was applied to ECG signals denoising. The advantage of wavelet approach is probability to obtain excellent quality signal for beat to beat. This paper aims to calculate fractal dimension (FD) to classify different cardiac diseases. The use of wavelets and fractal analysis tools are appropriated to the irregular signal analysis, because the characterization of the local regularity is important in the description of this signals. We use the neural networks to classification. The artificial neural networks have remarkable properties: adaptability, learning from examples and generalization, robustness against noise, they can be integrated into real-time process and they are remarkably effective for extracting rules from examples for problems that are difficult to modelable. Due to these properties, Connectionist methods have known these last years very successful in the analysis an interpretation of ECG signals.

\section{REFERENCES}

[1] Mendis S, Puska P, Norrving B. World Health Organization. Global atlas on cardiovascular disease prevention and control. Geneva: World Health Organization (2011).

[2] Kirk KJ, O'Shea J, Ruhf LK. ECG interpretation made incredibly easy!. Chris Burghardt.5 (2011).

[3] Sedielmaci I, Reguig FB. Detection of some heart diseases using fractal dimension and chaos theory. In 2013 8th International Workshop on Systems, Signal Processing and their Applications (WoSSPA) 1 (2013): 89-94.

[4] Oweis R, Hijazi L. A computer-aided ECG diagnostic tool. Computer methods and programs in biomedicine 3 (2006): 279-284.

[5] http://www.physionet.org/physiobank

[6] P. de Chazal, M. O’Dwyer and R. B. Reilly "Automatic Classification of Heartbeats Using ECG Morphology and Heartbeat Interval Features", IEEE Transactions on Biomedical Engineering, Vol. 51, Issue 7, pp: 1196-1206, July 2004.

[7] Mary HM, Singh D. Fractal dimension of electrocardiogram: distinguishing healthy and heartfailure patients. Journal of Electrocardiology 1 (2013): e21-e37

[8] QUAYE-BALLARD, J. A., OKRAH, T. M., ANDAM-AKORFUL, S. A., et al. Assessment of vegetation dynamics in Upper East Region of Ghana based on wavelet multi-resolution analysis. Modeling Earth Systems and Environment, 2020, vol. 6, no 3, p. 1783-1793.

[9] KAK, Subhash. Fractals with optimal information dimension. Circuits, Systems, and Signal Processing, 2021, p. 1-11.

[10] ISLAM, Nahina, HAMID, Nafiz Imtiaz Bin, MAHMUD, Adnan, et al. Detection of some major heart diseases using fractal analysis. International Journal of Biometrics and Bioinformatics (IJBB), 2010, vol. 4, no 2, p. 63.

[11]http://www.wahl.org/fe/HTML_version/link/FE4W/ c4.htm 
[12] Kania M, Fereniec M, Maniewski R. Wavelet denoising for multi-lead high resolution ECG signals. Measurement science review 4 (2007): 3033.

[13] Raj VN, Venkateswarlu T. ECG signal denoising using undecimated wavelet transform. In2011 3rd International Conference on Electronics Computer Technology 1 (2011): 94-98.

[14] Maghsoudi F, Kiani K. A powerful novel method for ECG signal de-noising using different thresholding and Dual Tree Complex Wavelet Transform. the 2th Int. Knowledge-Based Engineering and Innovation (2015).

[15] Donoho DL, Johnstone IM. Adapting to unknown smoothness via wavelet shrinkage. Journal of the american statistical association 1 (1995): 12001224.

[16] Kiani, Kourosh, and Farzane Maghsoudi. "Classification of 7 arrhythmias from ecg using fractal dimensions." Journal of Bioinformatics and Systems Biology 2.3 (2019): 53-65.

[17] HENDEL, Mounia, BENYETTOU, Abdelkader, et KHELIL, Hiba. Classification des Arythmies Cardiaques par les Réseau de Neurones Artificiels. In : The Fifth International Conference Sciences of Electronic, Technologies of Information and Telecommunications SETIT, Hammamet, Tunisia. 2009.

[18] JAMBUKIA, Shweta H., DABHI, Vipul K., et PRAJAPATI, Harshadkumar B. Classification of ECG signals using machine learning techniques: A survey. In: 2015 International Conference on Advances in Computer Engineering and Applications. IEEE, 2015. p. 714-721. 